\title{
Despertando Jovens Talentos com o Conhecimento da Computação
}

\author{
Dayanne Oliveira da Silva, Victor Ronchi Garcia, Igor Goulart de Oliveira, Daniela de \\ Freitas G. Trindade, Ederson Marcos Sgarbi, Luiz Fernando L. Nascimento
}

Centro de Ciências Tecnológicas - Universidade Estadual do Norte do Paraná (UENP) Campus Luiz Meneghel - Bandeirantes/ Pr

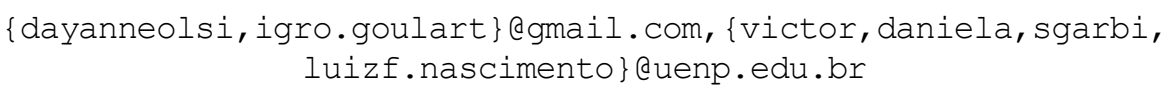

\begin{abstract}
Challenges have been imposed on the formation of computing professionals. Teaching programming is one of the great difficulties in computing courses education. In this sense, some researches demonstrate the importance of programming education from basic education to the formation of programming professionals. Thus, this research presents an experience with the application of workshops using the App Inventor tool in basic education of programming, experienced with third year of high school students in four schools in northern Parana region. This work was accomplished with the purpose of awakening young talents for computing area courses offered in the project operational area.
\end{abstract}

Resumo. Desafios têm sido impostos à formação de profissionais da área de computação. Ensinar programação é uma das grandes dificuldades na educação em cursos de computação. Neste sentido, pesquisas demonstram a importância do ensino de programação a partir do ensino básico para a formação destes profissionais. Assim, esta pesquisa apresenta uma experiência com a aplicação de oficinas utilizando a ferramenta App Inventor no ensino básico de programação, vivenciada com alunos de terceiro e segundo ano do ensino médio em quatro escolas da região Norte do Paraná. Este trabalho foi realizado com o intuito de despertar jovens talentos para os cursos da área de computação ofertados na região de atuação do projeto.

\section{Introdução}

O desenvolvimento da computação e o seu emprego nas atividades cotidianas mudaram a vida não só das empresas, mas, de todas as pessoas que as utilizam. Um "divisor de águas" no mundo da computação foi a chegada da internet. Este meio de comunicação alterou de forma ainda mais impactante o mundo. Alves (2006) comenta que a internet foi uma revolução digital, que mudou a maneira como as pessoas consomem informações.

Muitos foram os benefícios empregados pela internet e a computação, porém, é importante destacar também, os novos cargos que surgiram no século XXI, que demonstram um grande crescimento nesta área. Segundo a $\mathrm{BRQ}^{1}$ (2014) "O emprego relacionado à Tecnologia da Informação (TI) cresce em média $10 \%$ ao ano e já é responsável por 400.000 empregos de alta qualidade". Embora a demanda seja grande, ainda há uma escassez de profissionais qualificados na área de TI. Contribuindo para este cenário, verifica-se que os cursos na área de computação enfrentam dificuldades com relação ao preenchimento das vagas ofertadas e também com os altos índices de evasão.

${ }^{1}$ BRQ - Fundada em 1993, a BRQ é uma das maiores empresas de TI do país, segundo o Ranking DCI Brasil, 2012. 
Segundo os indicadores do Censo da Educação Superior de 2011 (INEP, 2012), apenas 52,7\% das vagas de Instituições de Ensino (IES) Privadas e Públicas são preenchidas. Sendo que o índice das IES Privadas é mais agravado, com apenas 47,3\% das vagas preenchidas. Ainda segundo o INEP (2012), para os cursos de ciência da computação, a evasão definitiva (considerando os alunos desvinculados, a transferência para outros cursos e os falecidos) representa algo em torno de $29 \%$ e a evasão temporária (considerando as matrículas trancadas), algo em torno de $20 \%$. Um dos grandes problemas enfrentados nesses cursos recai justamente na dificuldade com as disciplinas ligadas à programação. Grande parte dos estudantes apresenta dificuldade em entender os conceitos iniciais, aumentando, cada vez mais, o índice de evasão e reprovação nas disciplinas desta área (PRIETCH e PAZETO, 2010).

Face a este cenário, buscou-se investigar em alguns Colégios Estaduais da região do Norte Pioneiro, as dificuldades que os alunos possuem com as disciplinas ligadas à lógica matemática e programação e também promover oficinas de programação, a fim de despertar jovens talentos para a computação. No presente projeto, optou-se por trabalhar com a plataforma App Inventor (appinventor.mit.edu) por utilizar a programação em blocos tornando a aprendizagem mais significativa e atraente para os alunos. Além desses benefícios, o App Inventor permite abstrair a dificuldade dos códigos existentes nos paradigmas de programação tradicionais (GOMES e MELO, 2013). Esta ferramenta permite o desenvolvimento de aplicativos para smartphones que utilizam o sistema operacional Android, além disso, proporciona recursos para que tais aplicações utilizem funcionalidades dinâmicas de um smartphone Android, como por exemplo, câmera, GPS, giroscópio, dentre outras.

\section{O Estudo da Computação}

A computação trouxe mudanças significativas na forma como as pessoas trocam informações. É extremamente fácil pensar que a computação mudou só isso na vida das pessoas, no entanto, numa avaliação mais ampla, percebe-se que em quase todos os setores a computação está presente, melhorando cada vez a maneira como as pessoas realizam as atividades.

Santaella (2013) cita que "A Ciência da Computação estuda o modo como um computador está fundamentado como máquina, além de diversos tipos de problemas, cuja resolução pode utilizar a computação". Isso justifica o fato de que cada vez mais a computação está presente no dia-a-dia das pessoas. Reynol (2010) menciona que o uso da computação trouxe avanços significativos em pesquisas científicas nas diversas áreas do conhecimento, como, agronomia, astronomia e outras ciências.

A larga utilização da computação fez com que as pessoas também mudassem o estilo de vida, um exemplo foi com a chegada dos smartphones. Com um smartphone à mão, basta estar conectado à uma rede de internet para poder realizar inúmeras atividades mundo afora, sem sair do lugar. Porém, a programação de computadores, smartphones e demais dispositivos, não é uma tarefa trivial. Bittencourt et al. (2013) relata que o estudo da computação exige que o aluno desenvolva habilidades específicas de abstração e pensamento computacional.

Enfatizando a dificuldade em compreender o nível de abstração que requer a programação, Borges (2000) ressalta a importância de ambientes e softwares que auxiliem no aprendizado, um recurso a mais para auxiliar na prática e progredir no desenvolvimento exercitando a lógica. 
Pears et al. (2007) destaca que a programação de computadores é uma disciplina tradicionalmente difícil para estudantes iniciantes e que algumas pesquisas nesta área têm sido desenvolvidas, a fim de superar estas dificuldades. Para Raabe e Silva (2005) são várias as origens das dificuldades enfrentadas pelos alunos durante o processo de ensino aprendizagem de programação, como: a exigência lógico-matemático preeminente na disciplina, a dificuldade de apreensão, por parte do professor ou até mesmo o ritmo de aprendizagem de cada aluno.

Estudos demonstram que os índices de reprovação e evasão nas disciplinas de programação são bem altos. Robins (2010) destaca que fatores como, capacidade cognitiva, estilos cognitivos, motivação e atitude contribuem para este cenário. Segundo Pereira e Rapkiewicz (2004), com um número consideravelmente grande de desistências e reprovações em programação, é necessário viabilizar métodos, recursos e ferramentas diferenciadas para alcançar a aprendizagem e atrair mais pessoas para a área. Neste contexto, algumas ferramentas têm auxiliado no estudo da programação de maneira interativa e lúdica, como, Alice (COOPER, DANN e PAUSCH, 2000), Linguagem LOGO (PAPERT, 1985), Scratch (RESNICK, 2006) e AppInventor (WOLBER et al., 2014).

O Alice é um software pedagógico criado pelos pesquisadores da CMU. É um ambiente de programação que permite aos usuários, com pouca ou nenhuma experiência em computação, programar caracteres ou objetos em um mundo virtual, de forma muito parecida com um videogame (SHELLY et al., 2007). O Alice, inicialmente, foi desenvolvido para facilitar o teste de novas tecnologias e tornou-se uma ferramenta didática em cursos da área de computação (ZOTOVICI \& MENEZES, 2010).

A linguagem Logo é uma linguagem de programação para uso educacional, que foi desenvolvida no Massachusetts Institute of Technology (MIT pelo professor e matemático Seymour Papert. A linguagem logo foi instituída na década de 80 e segundo Papert (1985) surgiu como uma nova definição para a alfabetização, a "Alfabetização Tecnológica". A linguagem Logo estabelece, simultaneamente, uma teoria de aprendizagem, uma linguagem de comunicação e um conjunto de unidades materiais que permitem evidenciar os procedimentos mentais empregados por um indivíduo para resolver os problemas que lhe são apresentados.

As ferramentas Scratch e AppInventor, são ferramentas lúdicas que permitem montar estruturas por meio de blocos de comando, não necessitando o uso de linhas de códigos. Cada ferramenta possui suas particularidades e seus recursos voltados à uma atividade específica. No Scratch as funcionalidades presentes possibilitam ao usuário desenvolver animações e jogos, sua interface é amigável e possui um ambiente interativo que permite o desenvolvedor explorar sua criatividade. No AppInventor o usuário tem a possibilidade de adquirir a experiência de desenvolver aplicativos para o próprio smartphone, utilizando as funções nativas que estão contidas no sistema operacional Android como por exemplo o uso de banco de dados.

O Scratch foi desenvolvido pelo grupo de pesquisa do MIT Media Lab - o Lifelong Kindergarten Group (LLK). O Scratch apresenta uma linguagem de programação visual que permite a manipulação de mídias, tais como, imagens e músicas para a criação de histórias interativas, jogos, animações, dentre outros (Maloney et al. 2010). O AppInventor é uma ferramenta online desenvolvida pelo MIT - Massachusetts Institute of Technology em parceria com a Google Inc, que permite o desenvolvimento de aplicativos por meio da combinação de blocos de instruções, facilitando a criação para quem não tem o conhecimento de programação. 
V Congresso Brasileiro de Informática na Educação (CBIE 2016)

Anais do XXII Workshop de Informática na Escola (WIE 2016)

\section{Trabalhos Relacionados}

Souza (2013) descreve uma experiência adquirida em um curso de Introdução à Algoritmos no Ensino Médio em uma escola pública da região metropolitana de Belém Pará, oferecido pelos alunos do curso de Licenciatura em Computação por meio do Programa Institucional de Bolsas de Iniciação à Docência (PIBID). O curso ministrado teve como finalidade introduzir o raciocínio lógico utilizando algoritmos escritos em Portugol. Este trabalho teve o intuito de avaliar o grau de receptividade dos alunos do ensino médio e também de promover a aprendizagem e a vivência prática do ensino de de lógica e algoritmos pelos alunos da Licenciatura. $\mathrm{O}$ curso foi realizado no contra turno do ensino regular e teve duração de 40 horas. No total foram inscritos 34 alunos, no entanto, somente 5 alunos concluíram e se declararam motivados à fazer um curso superior na área de computação. Como trabalho futuro, o autor propõe aumentar a carga horária do curso para aprofundar os conceitos de algoritmos.

Marques et al. (2011), proporcionou aos alunos do ensino médio da região do Vale do Mamanguape da Paraíba a oportunidade de adquirir competências relacionadas ao desenvolvimento de jogos digitais utilizando Pyton. A escolha de tal linguagem foi defendida por descomplicar o aprendizado de algoritmos e programação. $\mathrm{O}$ desejo dos autores é pautado em motivar os alunos para cursar computação e cooperar na formação dos licenciados em computação. A oficina teve carga horária de 16 horas e contou com 16 participantes, que avaliaram como positiva a experiência, despertando o interesse pela área de computação. Como trabalho futuro, o projeto buscaria aplicar aulas adicionais a fim de incentivar os alunos a participar na modalidade de programação nas Olimpíadas Brasileiras de Informática.

Gomes et al. (2014), utilizaram a ferramenta Scratch para incentivar as meninas ao aprendizado em computação. A aplicação teve como público alvo alunas do primeiro ano do Ensino Médio de uma escola pública em Sergipe e objetivou o ensino de disciplinas da ciências exatas com o auxílio da ferramenta. O projeto possibilitou que as participantes criassem animações e documentos interativos envolvendo os conhecimentos em matemática, física e química. As alunas apresentaram um aproveitamento satisfatório e demonstraram-se motivadas à programação, conseguindo colocar em prática o que aprenderam por meio de atividades e projetos, os quais incentivaram o uso do raciocínio lógico.

O ensino de programação deve ser realizado de forma atrativa, segundo Finizola et al. (2014), utilizando recursos e técnicas que despertem o interesse, a fim de proporcionar à quem está tendo os primeiros contatos com a programação uma experiência positiva. Assim, neste trabalho, foi aplicado um curso utilizando a ferramenta App Inventor aos alunos do Ensino Médio pertencentes ao $1^{\circ}$ ano de uma escola pública estadual da cidade de Rio Tinto - PB. Os alunos puderam treinar a programação desenvolvendo aplicativos que poderiam ser executados em seus próprios dispositivos móveis para, então, terem a real noção de como os comandos reagem à forma como são dispostos. Todo o processo da construção do raciocínio, a busca por solução para determinado exercício foi trabalhado de modo dinâmico para que os alunos interagissem com a plataforma exercitando os conceitos de programação e obtendo resultados efetivos.

Guedes (2014) apresenta os resultados de um estudo observacional realizado na disciplina introdutória de programação em uma Universidade Pública Brasileira. A preocupação da autora é comparar o índice dos alunos novatos com os alunos de reprovação prévia. O estudo foi realizado com 147 acadêmicos e teve a duração de um semestre. Os resultados obtidos serão argumentos para utilizar o Pyton como linguagem de programação didática e também adotar novas estratégias de ensino. 
V Congresso Brasileiro de Informática na Educação (CBIE 2016)

Anais do XXII Workshop de Informática na Escola (WIE 2016)

\section{Metodologia}

Os procedimentos metodológicos utilizados para as oficinas de Introdução a Lógica de Programação com abordagem lúdica para despertar o interesse pela computação nos alunos do Ensino Médio foram: (a) Estudo das ferramentas Scratch e AppInventor 2; (b) Definição do plano de ensino para a realização das aulas; (c) Elaboração da apostila com o conteúdo programático, exemplos e exercícios, para ser utilizada como apoio nas aulas das oficinas; (d) Apresentação do Projeto e Seleção dos participantes, nos quatro Colégios Estaduais de Ensino Médio envolvidos, tendo como público alvo os alunos dos $3^{\circ} \mathrm{s}$ e $2^{\circ} \mathrm{s}$ anos; (e) Preparação dos Laboratórios de informática dos colégios para a execução das oficinas; (f) Aplicação de um questionário a fim de verificar o grau de conhecimento em computação dos alunos selecionados e identificar as potenciais dificuldades; (g) Aplicação das aulas com as turmas formadas a partir dos alunos selecionados em cada Colégio; (h) Estímulo ao raciocínio lógico e criatividade por meio de atividades que visavam a criação de pequenos projetos de programação, partindo de trabalhos individuais e em equipes; (i)Aplicação de questionário ao término das oficinas, a fim de comparar se houve acréscimo significativo no conhecimento sobre a computação; (j) Premiação para os alunos que se destacaram durante as oficinas e Realização da maratona de programação com os alunos que foram destaque durante as oficinas, para que pudessem colocar em prática os conhecimentos adquiridos.

\section{Desenvolvimento das Oficinas}

Para o desenvolvimento das oficinas foram escolhidas as ferramentas Scratch, para a introdução dos conceitos de programação e AppInventor2, para o aprofundamento do tema, voltado ao desenvolvimento de aplicações. Estas ferramentas foram escolhidas por apresentarem uma interface de fácil manuseio, vários recursos e componentes para o desenvolvimento de programas, animações e jogos.

Outra pesquisa, conforme mencionado na seção 3, apresentou um estudo com a ferramenta AppInventor, porém, o estudo proposto por Finizola et al. (2014) se deu em uma turma de 20 alunos e com metodologia diferente, em que os conceitos de programação foram introduzidos diretamente pelo AppInventor.

Já no presente trabalho, os conceitos básicos de programação foram introduzidos, primeiramente, pelo estudo de pequenos algoritmos e fluxogramas e posteriormente pelo uso do AppInventor. Os conteúdos foram divididos em duas etapas: (i) noções básicas de programação com a criação de fluxogramas e pequenos algoritmos, (ii) utilização de ferramentas lúdicas para o desenvolvimento de aplicações. Os colégios participantes foram: Colégio Estadual Rui Barbosa de Abatiá/PR; Colégio Estadual Durval Ramos Filho de Andirá/PR; Colégio Estadual Marcílio Dias de Itambaracá/PR; e Colégio Estadual Mailon Medeiros de Bandeirantes/PR. Em cada Colégio a oficina foi realizada com um total de 9 aulas, com 2,5 horas de duração cada; As aulas foram realizadas nos laboratórios de informática, dos próprios colégios

As primeiras visitas realizadas nos colégios tinham o intuito de apresentar o projeto "Despertando Jovens Talentos com o Conhecimento da Computação" à equipe administrativa e pedagógica, verificar as possibilidades de datas e horários de aplicação das oficinas e preparar os laboratórios para as aulas. Vale ressaltar que todo o processo para a execução da oficina foi realizado em um colégio por vez, seguindo uma sequência definida pela melhor logística de visita às cidades comtempladas.

A partir destes primeiros contatos, foi programada a apresentação do projeto nos referidos colégios, com uma breve introdução do que seria trabalhado, para os alunos dos $3^{\circ} \mathrm{s}$ e $2^{\circ} \mathrm{s}$ anos com o propósito de convidá-los e motivá-los a participarem das oficinas no período vespertino. Neste momento, tido como um dos mais importantes, uma vez que 
era preciso despertar o interesse dos alunos pela oficina de programação, buscou-se estimular os alunos com demonstrações dos programas que seriam utilizados e diversas possibilidades de criação de jogos e aplicativos.

Houve grande interesse dos alunos, sendo necessário abrir turmas extras em dois colégios. No total foram 125 participantes, divididos da seguida forma: 45 alunos no Colégio Estadual Rui Barbosa; 21 alunos no Colégio Estadual Durval Ramos Filho; 39 alunos no Colégio Estadual Marcílio Dias e 20 alunos no Colégio Mailon Medeiros.

Na primeira aula, em cada colégio, foi aplicado o questionário de diagnóstico inicial com 10 perguntas voltadas ao conhecimento básico de lógica e programação. Esta análise tinha a finalidade de identificar se os alunos possuíam algum conhecimento prévio, ou mesmo, ideia do assunto. Após análise realizada em todos os colégios, foi constatado que apenas $13,60 \%$ tiveram contato com a programação antes do projeto. Observou-se também que a maioria dos alunos tinha a impressão de que a programação era algo muito difícil e inacessível.

Ainda nesta primeira aula, os alunos foram perguntados sobre os cursos de informática da região. Para a surpresa, a maioria dos alunos desconhecia os cursos na área de computação ofertados pela universidade mais próxima, a UENP, e, também, que a universidade era pública, portanto, gratuita. Após este primeiro contato, com a aplicação do questionário, foi passado o vídeo "The hour of code is here" (code.org), para uma breve exposição da importância da programação nos dias atuais e como uma forma de motivar os alunos para a participação nas oficinas e construção de seus próprios programas.

O conteúdo da apostila começou a ser aplicado, seguindo a sequência préestabelecida nos Planos de Aula. Assim, foram introduzidos os conceitos de algoritmos, processos e fluxogramas. Na sequência, os algoritmos foram estudados na prática, para que os alunos pudessem compreender a sua aplicação e realizar os exercícios de construção de algoritmos de baixa complexidade. A distribuição do Conteúdo em semanas por Colégio seguiu o cronograma representado na Tabela 1.

Tabela 1 - Cronograma de conteúdos

\begin{tabular}{|l|c|c|c|c|c|}
\hline Conteúdo/Semana & $\mathbf{1}^{\mathbf{a}}$ semana & $\mathbf{2}^{\mathbf{a}}$ semana & $\mathbf{3}^{\mathbf{a}}$ semana & $\mathbf{4}^{\mathbf{a}}$ semana & $\mathbf{5}^{\mathbf{a}}$ semana \\
\hline Algoritmos e Processos & $\mathrm{X}$ & & & & \\
\hline Fluxogramas & $\mathrm{X}$ & & & & \\
\hline Scratch & & $\mathrm{X}$ & $\mathrm{X}$ & $\mathrm{X}$ & \\
\hline AppInventor 2 & & $\mathrm{X}$ & $\mathrm{X}$ & $\mathrm{X}$ \\
\hline
\end{tabular}

O ensino do fluxograma teve como objetivo a construção da sequência lógica, a organização do raciocínio e a representação gráfica do passo a passo de execução de um processo, buscando facilitar a visualização e compreensão. A escrita de algoritmos possibilitou esquematizar a lógica para a solução de problemas e execução de tarefas. As estruturas de seleção, repetição, condição foram apresentadas nessa fase.

Ao iniciar o treinamento com as ferramentas Scratch e AppInventor 2, os alunos não apresentaram grandes dificuldades. Já no primeiro contato conseguiram desenvolver pequenos programas envolvendo as operações aritméticas. Os primeiros programas foram elaborados junto com toda a turma a fim de explanar os comandos e para que os alunos se habituassem à interface das ferramentas. Assim, tiveram um passo a passo da lógica usada que está disponível na apostila de apoio. A Figura 1 apresenta duas imagens de uma das aulas do projeto. 


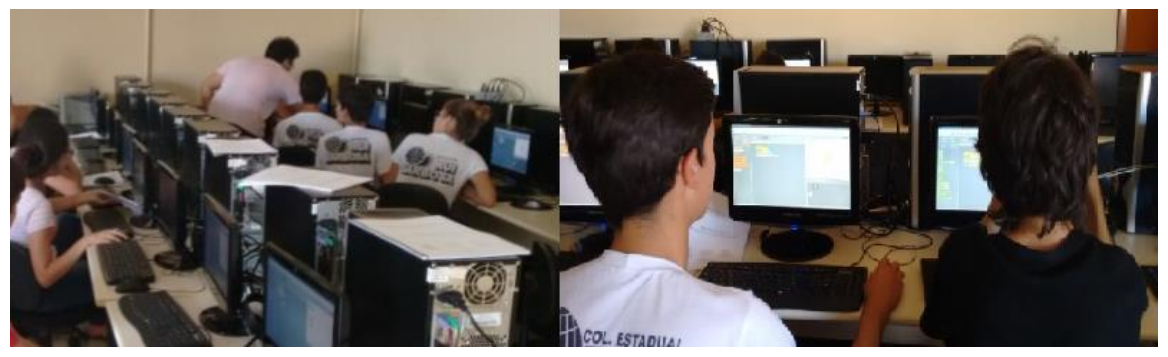

Figura 1 - Aplicação da oficina de programação

Listas de exercícios foram propostas e suas resoluções foram cobradas levando em consideração o tempo de entendimento de cada aluno. Desta forma, neste momento, foi dada atenção individual, buscando esclarecer as dúvidas a respeito das ferramentas. Além dos exercícios propostos em aula, os participantes dispuseram de um tempo para explorar as ferramentas e propor novos projetos. Assim, puderam, ao mesmo tempo, executar o programa que desejassem e se familiarizar com a programação.

\subsection{Avaliação dos Resultados}

Ao final das oficinas, foi realizada a aplicação de outro questionário, que contribuiu para a análise do aproveitamento dos alunos participantes. Para aferir os percentuais de aproveitamento foram utilizados os dados da avaliação final e também os dados referentes à execução dos exercícios e desafios propostos durante as aulas da oficina. Na Figura 2 é apresentado o número de alunos que obtiveram aproveitamento acima de $90 \%$; de $80 \%$ a $89 \%$; de $70 \%$ a $79 \%$; de $60 \%$ a $69 \%$; de $50 \%$ a $59 \%$ e abaixo de $50 \%$. Verifica-se pelos dados, que a maioria - aproximadamente $63 \%$ dos alunos, obtiveram aproveitamento superior a $70 \%$ e apenas $10 \%$ aproximadamente obtiveram aproveitamento abaixo de $50 \%$, o que indica um resultado positivo.

Ao final da oficina, para cada colégio foram premiados os três alunos que obtiveram o melhor desempenho. O primeiro lugar foi premiado com um Tablet $\mathrm{e}$ medalha de ouro, para o segundo e o terceiro lugar, respectivamente, medalhas de prata e bronze.

\section{Aproveitamento da oficina de programação}

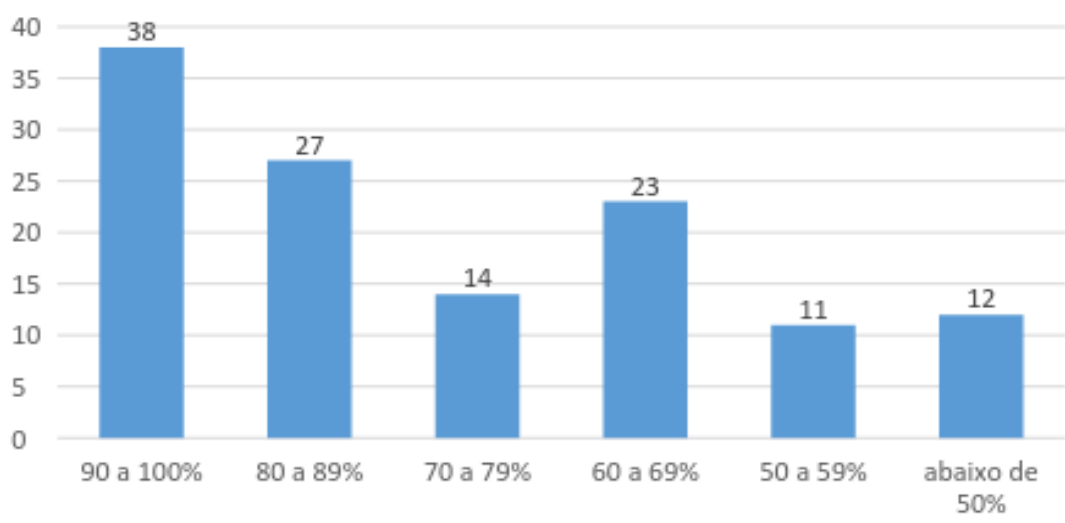

Figura 2: Gráfico com aproveitamento nas Oficinas de Programação

Os alunos participantes do projeto visitaram a UENP no dia da premiação. Em um primeiro momento, os alunos conheceram as instalações do Centro de Ciências Tecnológicas que abriga os cursos de Sistemas de Informação (Bacharel e Licenciatura Noturno) e Ciência da Computação (Bacharel - Integral). Na sequência, foi ministrada uma palestra, por um docente da UENP, que relatou a diferença entre os cursos de Sistemas de Informação e Ciência da Computação. Também foi apresentado um vídeo institucional, no qual foram apresentados todos os cursos da UENP. 
Ao fim da visita, para cada colégio foi realizada uma pesquisa sobre o interesse dos participantes da oficina em ingressar em cursos da área de computação. Observou-se que pelo menos $67,2 \%$ dos participantes das oficinas demonstraram interesse por cursos da área de computação. Positivamente, pode-se destacar que os alunos que não relataram interesse são os que obtiveram os piores desempenho no curso. Assim, preliminarmente, este resultado pode indicar que a falta de interesse desses alunos está vinculada a falta de vocação para a área.

Para uma a análise e discussão destes resultados da pesquisa, foi comparada a quantidade de alunos matriculados até 2015 nos cursos do Centro de Ciências Tecnológicas da UENP (Sistemas de Informação e Ciência da Computação), fornecidos pela Secretaria Acadêmica (Tabela 2).

Tabela 2. Origem dos alunos matriculados nos cursos do CCT-UENP

\begin{tabular}{|l|c|c|c|c|}
\hline \multirow{2}{*}{ Cidades de Origem } & \multicolumn{4}{|c|}{ Quantidade e Percentual de Alunos Matriculados } \\
\cline { 2 - 5 } & $\begin{array}{l}\text { Ciência da } \\
\text { Computação }\end{array}$ & $\begin{array}{l}\text { \% Ciência da } \\
\text { Computação }\end{array}$ & $\begin{array}{l}\text { Sistemas de } \\
\text { Informaçáo }\end{array}$ & $\begin{array}{l}\text { \% Sistemas de } \\
\text { Informação }\end{array}$ \\
\cline { 2 - 5 } Itambaracá & 24 & $29 \%$ & 75 & $45 \%$ \\
\hline Demais cidades do Paraná & 38 & $46 \%$ & 60 & $36 \%$ \\
\hline Estado de São Paulo & 21 & $25 \%$ & $\mathbf{1 6 7}$ & $19 \%$ \\
\hline TOTAL & $\mathbf{8 3}$ & $\mathbf{1 0 0 \%}$ & $\mathbf{1 0 0 \%}$ \\
\hline
\end{tabular}

A partir destes dados observa-se que: (i) No curso de Sistemas de Informação (SI), aproximadamente $45 \%$ dos alunos pertencem às cidades de origem dos colégios participantes do projeto. Verifica-se que, pelo curso de SI ser noturno, o perfil dos alunos é diferenciado, agregando, normalmente, pessoas que trabalham e estudam. Desta forma, os alunos são oriundos de cidades mais próximas ao local do curso; (ii) No curso de Ciência da Computação (CC), 29\% dos alunos pertencem às cidades de origem dos colégios participantes do projeto. Por ser integral, o curso de CC apresenta um perfil diferenciado de alunos, recebendo alunos de cidades mais distantes que passam a residir no local do curso.

Analisando o número de alunos matriculados até 2015 (Tabela 2), que representam os matriculados em quatro anos de curso (correspondente ao $1^{\circ}, 2^{\circ}, 3^{\circ}$ e $4^{\circ}$ ano), temos um total de 99 alunos matriculados nos cursos de SI e CC. Assim, considerando que o número total de participantes do projeto foi de 125 e que $67,2 \%$ ( 84 alunos) demonstraram interesse em ingressar nos cursos da área de computação, entendese que os resultados foram positivos. Observando o alto número de interessados em ingressar em cursos de computação (84 alunos), a partir da aplicação do projeto em apenas 1 colégio para cada uma das 4 cidades contempladas, verifica-se o potencial das oficinas na divulgação e no despertar de interesse para a área.

\section{Considerações Finais}

A metodologia utilizada no presente trabalho permitiu aos alunos terem uma visão geral das diferentes formas de programação. Os conceitos inicias serviram para embasar o conhecimento a respeito do processo manual de codificação e também pelo uso de ferramenta gráfica orientada ao design. Desta forma, os alunos foram apresentados ao mundo real da programação, com as diversas nuances, para que compreendessem a real complexidade, mas, para que também despertassem o interesse pela área.

A ferramenta Scratch serviu para que os alunos, inicialmente, pudessem visualizar a materialização de um programa para, posteriormente, avançarem na programação para Android. $\mathrm{O}$ fato de terem usado uma ferramenta de programação para smartphone contribuiu para a motivação dos alunos, uma vez que quase todos possuíam um smartphone com sistema operacional Android. 
Por fim, como trabalho complementar, está em andamento a organização de uma maratona de programação básica juntando todos os colégios em que as oficinas foram aplicadas, como forma de estimular os participantes ao contínuo desenvolvimento de suas habilidades na programação. As atividades da maratona constarão de jogos de lógica e desenvolvimento de 5 programas para Scratch, para serem executados em trios, com o tempo limite de 4 horas para a resolução dos exercícios.

\section{Agradecimentos}

O presente trabalho foi realizado com o apoio da Universidade Sem Fronteiras pelo fomento ao projeto "Despertando Jovens Talentos com o Conhecimento da Computação". Também ficam aqui os agradecimentos às escolas que foram receptivas e demonstraram total apoio para tornar possível o desenvolvimento de tais atividades.

\section{Referências}

Alves, R. C. (2006) “Comunicação e Sociedade”, vol. 9-10, pp. 93-102.

Bittencourt, R. A., Rocha, A. S., Santana, B. L., Santana, C. S., Carneiro, D. A., Borges, G. A., Chalegre, H. S., Silva, J. F. J., Santos, J. M. J., Silva, L. A. e Andrade, P. H. M. O. (2013) "Aprendizagem de Programação Através de Ambientes Lúdicos em um Curso de Engenharia de Computação: Uma Primeira Incursão", Em: XXI WEI Workshop sobre Educação em Computação, Maceió, AL, Brasil, p. 749 - 758.

Borges, M. A. F. (2000) "Avaliação de uma Metodologia Alternativa para a Aprendizagem de Programação", Em: Congresso da Sociedade Brasileira de Computação. Florianópolis, SC, Brasil.

BRQ. "Carreira em TI”, http://www.brq.com/carreira-em-ti, Outubro.

Cooper, S., Dann, W. e Pausch, R. (2000) "Making the connection: Programming with animated small worlds", Em: Proceedings of the 5th annual SIGCSE/SIGCUE ITiCSE conference on Innovation and technology in computer science education (Helsinki, Finland), ITiCSE '00, ACM, New York, NY, 41-44.

Finizola A. B., Raposo E. H. S., Pereira M. B. P. N. P., Gomes W. S. G. e Araújo A. L. S., Souza F. V. C. (2014) "O ensino de programação para dispositivos móveis utilizando o MIT-App Inventor com alunos do ensino médio", Em: XX WIE Workshop de Informática na Escola, Dourados, MS, Brasil.

Gomes W. F., Louzada C.S., Nunes M.A.N., Salgueiro E. M. e Andrade B. T., (2014) "Incentivando meninas do ensino médio à área de Ciência da Computação usando o Scratch como ferramenta", Em: XX WIE - Workshop de Informática na Escola, Dourados, MS, Brasil, Anais do III Congresso Brasileiro de Informática na Educação.

Gomes, T. C. S. e Melo, J. C. B. (2013) "App Inventor for Android: Uma Nova Possibilidade para o Ensino de Lógica de Programação", Em: Congresso Brasileiro de Informática na Escola, Campinas, SP, Brasil.

Guedes, E. B.; (2014), "Um Estudo Observacional sobre a Disciplina Introdutória de Programação", Em: XX WIE - Workshop de Informática na Escola, Dourados, MS, Brasil, Anais do III Congresso Brasileiro de Informática na Educação.

Maloney, J., Resnick, M., Rusk, N., Silverman, B. e Eastmond, E. (2010) "The scratch programming language and environment", Em: Transactions on Computing Education, ACM, vol. 10, n. 4, article 16, 15 pages.

Marques, D. L., Costa, L. F. S., Silva, M. A. A. e Rebouças A. D. D. S. (2011) “Atraindo Alunos do Ensino Médio para a Computação: Uma Experiência Prática de Introdução a Programação utilizando Jogos e Python", Em: XVII WIE - Workshop de Informática na Escola, Aracajú, SE, Brasil, Anais do XXII Simpósio Brasileiro de Informática na 
V Congresso Brasileiro de Informática na Educação (CBIE 2016)

Anais do XXII Workshop de Informática na Escola (WIE 2016)

Educação.

Papert, S. (1985) "Logo History" http://el.media.mit.edu/logofoundation/what is logo/history.html, Maio.

Pears, A., Seidman, S., Malmi, L., Mannila, L. Adams, E. Bennedsen, J., Devlin, M. e Paterson, J. (2007) "A survey of literature on the teaching of introductory programming", Em: Working group reports on ITiCSE on Innovation and technology in computer science education, ITiCSE-WGR '07, ACM , New York, NY, USA , 204223.

Pereira, J. J. C. R. e Rapkiewicz, C. E. (2004) "O processo de Ensino e Aprendizagem de Algoritmos e Programação: Uma Visão Crítica da Literatura”, Em: III WEIMIG Workshop de Educação em Computação e Informática, Belo Horizonte, MG, Brasil.

Pinheiro, C. D. B., Leal, S. D. A. e Lima, C. P. (2015) "Um Cenário Viável para Motivar os Alunos do Ensino Fundamental para um Futuro Ingresso nos Cursos Superiores de Computação", Em: XXI WIE - Workshop de Informática na Escola, Maceió, AL, Brasil.

Prietch, S. S., Pazeto, T. A. (2010), "Mapeamento de Cursos de Licenciatura em Computação seguido de Proposta de Padronização de Matriz Curricular", Em: WEI XVIII Workshop sobre Educação em Computação, Belo Horizonte, MG, Brasil.

Raabe, A. L. A. e Silva, J. M. C. (2005) "Um Ambiente para Atendimento as Dificuldades de Aprendizagem de Algoritmos", Em: XXV CSBC - Congresso da Sociedade Brasileira de Computação. São Leopoldo, RS, Brasil.

Resnick, M. (2006) "Scratch: Democratizar a Expressão Digital”, http://web.media.mit.edu/ mres, Abril.

Reynol, F. "Encontro discute a importância da computação para a ciência. Agência Fapesp", http://www.inovacaotecnologica.com.br/noticias, Abril.

Robins, A. (2010) "Learning edge momentum: A new account of outcomes in CS1", Computer Science Education, v. 20, n. 1, p. 37-71.

Santaela, L., Gala, A., Policarpo, C. e Gazoni, R. (2013) "Desvelando a internet das coisas", Em: Revista Geminis.

Scaico, P. D., Corlett, E. F., Paiva L. F., Raposo, E. H. S. e Alencar Y. (2012) "Relato da Utilização de uma Metodologia de Trabalho para o Ensino de Ciência da Computação no Ensino Médio", Em: XVIII WIE - Workshop de Informática na Escola. Rio de Janeiro, RJ, Brasil.

Scratch "Crie estórias, jogos e animações: Partilhe com gente de todo o mundo", http://www.scratch.mit.edu, Maio.

Shelly, G. B., Cashman, T. J. e Hebert, C. W. (2007) Em: "Introdutory Concepts and Techniques”, Em: Shelly Cashman Series ${ }^{\circledR}$, Alice 2.0, Course Technology, Cengage Learning, Boston Massachusetts, USA, (Printed in China).

Sousa, D. F. (2013) “Desenvolvendo a Lógica e Algoritmos no Ensino Médio”, Em: XIX WIE - Workshop de Informática na Escola. Campinas, SP, Brasil.

Wolber, D., Abelson, H., Spertus, E. e Lonney, L. (2014) Em: “AppInventor 2: Create Your Own Android Apps", O’Relly Media, Inc., 1005 Gravenstein Highway North, Sebastopol, CA 95472.

Zotovici, A., Menezes, C. E. D. (2010) "Tutorial para lógica de programação usando ALICE", Anais do Congresso Alice Brasil. 